\title{
CONTINUUM MODELS FOR ELECTRICAL BREAKDOWN IN PHOTOCONDUCTIVE SEMICONDUCTOR SWITCHES ${ }^{1}$
}

\author{
H. P. Hjalmarson ${ }^{\xi}$ and K. Kambour \\ Sandia National Laboratories, PO Box 5800 \\ Albuquerque, New Mexico, 87185 USA \\ C. W. Myles \\ Department of Physics \\ Texas Tech University \\ Lubbock, Texas, 79409 USA \\ R. P. Joshi \\ Department of Electrical and Computer Engineering \\ Old Dominion University \\ Norfolk, Virginnia 23529 USA
}

\begin{abstract}
In this paper, continuum models for electrical breakdown are described. These models are based on calculations of the carrier distribution function as a function of electric field and carrier density. In these continuum models, the impact ionization rate is approximated in the extremes of low and high carrier density. These continuum models are used for calculations of electrical breakdown in GaAs. In particular, these models are applied to the operation of photoconductive semiconductor switch (PCSS) devices. This comparison with data suggests that a new physics mechanism is required to explain the PCSS data, a new mechanism is hypothesized.
\end{abstract}

\section{I.INTRODUCTION}

Electrical breakdown of an insulator occurs if the applied electric field exceeds a threshold field, called the breakdown field. At fields above the breakdown field, the electric current grows dramatically. This electrical current heats the insulator, and it can lead to the destruction of the material.

Much effort over many years has been focused on understanding electric breakdown in solid insulators [1]. An important theoretical foundation was the avalanche breakdown theory formulated for the breakdown of insulating gases [2]. In this theory, applied to solids, an initial electron is heated by the field until it has achieved enough energy to create another electron by impact ionization. The Joule heating of this electron by the field competes with cooling by phonon emission. Assuming that the electrode separation is large enough to allow a carrier to repeatedly gain enough energy from the electric field to reach the impact ionization threshold (this energy is approximately the band-gap of the material above the conduction band minimum), this process eventually leads to an "avalanche" of electron-hole pairs.

Extensive experimental work has focused on determining the breakdown field for several insulators [1]. This idealized characteristic has been elusive because electric breakdown at a lower field may be triggered by defects in the sample. Furthermore, breakdown is a probabilistic process even for an ideal sample because it is triggered by random fluctuations in the carrier density.

A type of low field electrical breakdown has been observed in GaAs and InP during the development of optically-triggered photoconductive semiconductor switches (PCSS's) [3,4]. This breakdown is triggered by optically injecting carriers In this case, the breakdown is triggered into a steady state condition in which the carrier density, carrier temperature, and the electric field remain approximately constant $[3,4]$. In this situation, called the "lock-on" effect, the current flows in filaments, and is sustained by a field, called the "lock-on" field, that is much lower than the intrinsic breakdown field.

In this paper we focus on development of a robust continuum mechanism to be used for time-dependent calculations of electrical breakdown and lock-on in a sample that is connected to an electrical circuit. This mechanism is based on the results from microscopic calculations.

\section{II.THEORY}

A. Qualitative Physics:

\footnotetext{
${ }^{1}$ Sandia is a multiprogram laboratory operated by Sandia Corporation, a Lockheed Martin company, for the United States Department of Energy under contract DE-AC04-94AL85000.

६ email: hphjalm@sandia.gov
} 
In earlier work, we developed a theory, collective impact ionization, to explain lock-on.[7,8,9] In this theory, the approach taken is to formulate a mechanism whereby two steady state solutions exist for each electric field. In this way, the theory can produce solutions for which the current flows in filaments. Such filamentary current flow can be regarded as the coexistence of a high current solution inside the filament and a low current solution outside the filament.

This approach led to a focus on carrier-carrier scattering on the impact ionization process, the fundamental mechanism of electrical breakdown. Impact ionization is governed by the carrier distribution function at an energy approximately one band-gap above the conduction band edge or one band-gap below the valence band-edge. This impact ionization rate has been the focus of numerous previous investigations.

The distribution function depends on the competition between Joule heating of the carriers and the phonon emission cooling of the carriers.

To understand the mechanism, one can first consider the rate at a very high carrier density. In this case, one may assume that carrier-carrier scattering has produced a Maxwellian distribution function. Such a distribution can be characterized by its temperature, the carrier temperature.

The low density distribution function has a different appearance at the energies required for impact ionization. For this distribution, there are proportionately fewer carriers at any given energy because phonon scattering controls the shape of the distribution function. Consequently, for a given electric field, the low-density distribution function will lead to much less impact ionization.

At this point one can understand that this carrier-carrier scattering mechanism is consistent with filaments. Inside the filament, the large density leads to a Maxwellian distribution whose large impact ionization rate is consistent with the high carrier density. Outside the filament, the low carrier density will lead to a nonMaxwellian distribution whose low impact ionization rate is consistent with a low carrier density.

\section{B. Framework for Electrical Breakdown Calculations}

The basis for our calculations is an expression for the time rate of change of carriers, either electrons or holes. Focusing on electrons and ignoring gradients in carrier current, the resultant kinetic equation is

$$
\frac{d n}{d t}=R(n, F) n
$$

with

$$
R(n, F)=\sum f(k)\left\{r_{i i}-r_{\text {Auger }}-r_{\text {defects }}\right\} .
$$

In these expressions, $f(\mathbf{k})$ is the carrier distribution function, $\mathbf{k}$ is the wavevector, $n$ is the carrier density and $F$ is the electric field. The growth-decay rate
$R(n, F)$ contains contributions from the mechanisms that govern electrical breakdown. In agreement with previous work on electrical breakdown, the key growth mechanism is impact ionization. The reverse mechanism, Auger recombination is also included. Finally, recombination at defects is also included.

This quantity $R(n, F)$ is very important in our approach to electrical breakdown. The qualitative features of $R(n, F)$ can be used to understand electrical breakdown. In earlier work it was computed for various materials. As will be further discussed, this quantity will be used for continuum calculations.

Before focusing on continuum models, we examine the features contained in growth-decay rate $R(n, F)$. As implied in the earlier discussion, our approach focuses on the steady state solutions. These solutions are given by solving for the $n$ and $F$ pairs that correspond to the condition $R(n, F)=0$.

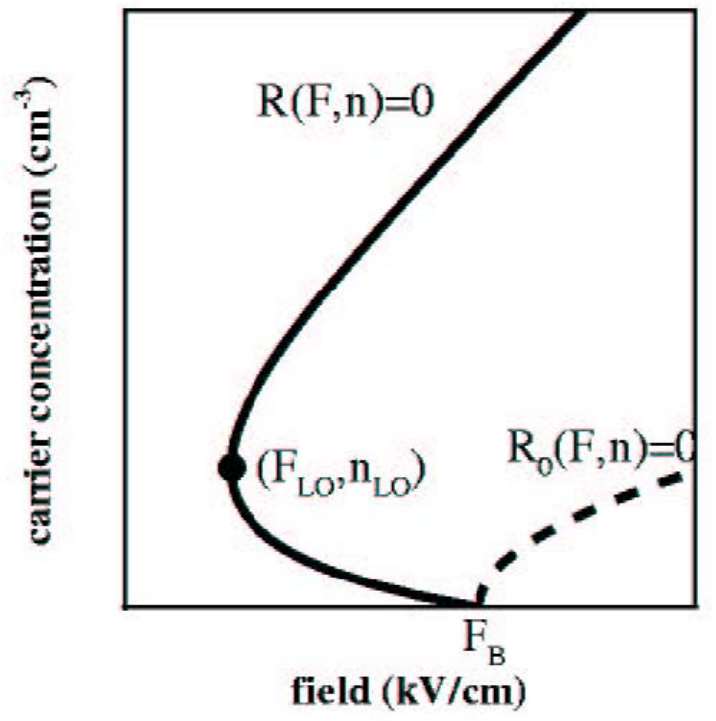

Figure 1 Plots of $R_{0}(n, F)=0$ (dashed curve) and $R(n, F)=0$ (solid curve).

To illustrate, we first ignore carrier-carrier scattering. The solution $R_{0}(n, F)=0$ is shown in Fig. 1. If the field is less than the avalanche breakdown field $F_{B}$, carrier growth will not occur because $R_{0}(n, F)<0$. However, if the breakdown field is exceeded, the carrier density will grow. In this case, the carrier density will grow until it reaches the density that lies on the $R$-curve. However, for these cases, only one solution is allowed. Thus no spatial dependence is allowed, and the breakdown will be uniform.

To include carrier-carrier scattering, the simplest approximation is to include the first-order term, the linear dependence on carrier density. The solutions for this case are also shown in Fig. 1.

Having included carrier-carrier, the other curve in Fig. 1 shows that two steady-state solutions are allowed for 
each electrical field. For example, at a given field less than the breakdown field, there are three allowed solutions if we include the zero density solutions.

This figure can be used to understand some qualitative features expected for a switch in a circuit. Although not considered in this paper, the qualitative features and stability of these solutions can be examined. A stability analysis can be done to show that for a simple circuit a stable solution will correspond to the lowest field. Inside the filament, the carrier density will be $n_{L O}$. For a given point on the circuit current-voltage curve of the switch in a circuit, the density in the filament will remain constant and the area of the filament will accommodate the current. The current-voltage curve will be nearly vertical between a minimum current determined by the minimum size of a filament and a maximum current determined by the area of the device. The minimum size will be determined by the diffusion length of the carriers.

\section{Direct k-space Calculations}

The quantity $R(n, F)$ can be calculated for specific materials. This has been done for $\mathrm{GaAs}, \mathrm{GaP}, \mathrm{InP}$ and $\mathrm{Si}$ $[7,8]$. For these calculations, the steady-state k-space distribution function $f(\mathbf{k})$ was calculated as a function of carrier density and electric field using a Monte Carlo method. This distribution function was then used to compute the rate $R(n, F)$. Furthermore, additional calculations were performed to obtain the curve $R(n, F)=0$. These calculations showed that this mechanism will lead to multiple steady state solutions at a given electric field.

\section{Continuum calculations and models}

The time-dependent continuum calculations focus on the transport of electrons and holes during electrical breakdown [10]. The calculations focus on kinetic equations for electrons and holes. These equations are governed by contribution from the electrical current and also carrier generation and recombination. The equations have the form:

$$
\begin{aligned}
& \frac{\partial n}{\partial t}=\frac{1}{q} \nabla \mathbf{J}_{n}+R_{n} \\
& \frac{\partial p}{\partial t}=-\frac{1}{q} \nabla \mathbf{J}_{p}+R_{p}
\end{aligned}
$$

In these equations, the currents are

$$
\begin{aligned}
& \mathbf{J}_{n}=-q v_{n} n+D_{n} \nabla n \\
& \mathbf{J}_{p}=q v_{p} p-D_{p} \nabla p
\end{aligned}
$$

These are the usual drift and diffusion contributions to the currents. The generation recombination terms are the quantities $R(n, F)$ for electrons and holes.

The carrier temperatures are also computed. The kinetic equations are the following.

$$
\begin{aligned}
& \frac{3 k_{B}}{2} \frac{\partial T_{n}}{\partial t}=-q \mathbf{v}_{\mathbf{n}} \cdot \mathbf{E}-E_{g} R_{i i A n}-R_{p n}\left(T_{n}, T_{L}\right) \\
& \frac{3 k_{B}}{2} \frac{\partial T_{p}}{\partial t}=q \mathbf{v}_{p} \cdot \mathbf{E}-E_{g} R_{i i A p}-R_{p p}\left(T_{p}, T_{L}\right) .
\end{aligned}
$$

The contributions in each equation, respectively, are Joule heating, impact ionization cooling, Auger heating and phonon cooling.

One important consideration for the carrier temperature equations is the inclusion of the effects of the impact ionization and Auger mechanisms. The impact ionization mechanism cools the carriers because a hot carrier with approximately a band-gap of energy is removed. This process creates an electron and a hole whose energies are both small. For similar reasons, Auger recombination heats the carriers.

To perform the calculations, an approximate continuum model is needed for the quantity $R(n, F)$. In a previous paper, one such model has been described and used [10]. In this paper, a new continuum model is described.

To make an approximate model, the key consideration is the impact ionization rate. For condensed matter applications, the combined contributions from impact ionization and Auger recombination can be written as

$$
\begin{aligned}
& R_{i i A n}=c_{n}\left(T_{n}\right)\left(n_{i}^{2}\left(T_{t n}\right)-n p\right) \\
& R_{i i A p}=c_{p}\left(T_{p}\right)\left(n_{i}^{2}\left(T_{t p}\right)-n p\right)
\end{aligned}
$$

In these expressions, $n_{i}(T)$ is the intrinsic carrier density:

$$
n_{i}(T)=\sqrt{N_{c}(T) N_{v}(T)} \exp \left(-E_{G} / k_{B} T\right)
$$

in terms of the conduction and valence band thermal density of states and carrier temperature.

The above expression represents the rate in the high density limit for which carrier temperature is a valid characteristic. At low carrier density, the impact ionization rate is much reduced. This effect is included by simply assuming that the carrier temperature is reduced at the high energy region of the distribution function that controls impact ionization.

The problems with this mechanism have been exposed in a recent set of calculations. First, the reduced temperature makes it impossible to achieve an equilibrium temperature at low fields. This was never a problem in electrical breakdown simulations but it arises if the field is very low.

\section{E. A New Continuum Model}

For this paper a new approach was taken. In this mechanism, the low density II rate is taken to be reduced relative to the rate in the high density limit. This produces a rate that has a field-dependence very similar to that found in avalanche breakdown experiments. Such experiments are focused on the low density limit of impact ionization.

This new mechanism is a conceptual improvement over the previous continuum mechanism. The predicted 
equilibrium carrier densities are correct and the calculations are easier and faster.

\section{DISCUSSION}

The calculations discussed in this paper are focused on GaAs. The GaAs band-structure used for these calculations is shown in Fig. 2. The resultant R-curves are shown in Fig. 3.

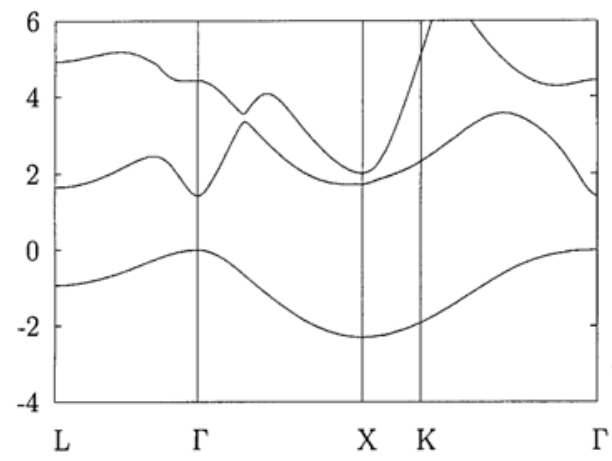

Figure 2 GaAs bandstructure.

The R-curve for the two continuum mechanisms applied to GaAs are shown in Fig. 3. By inspection, these curves have a similar shape but they differ in the details.

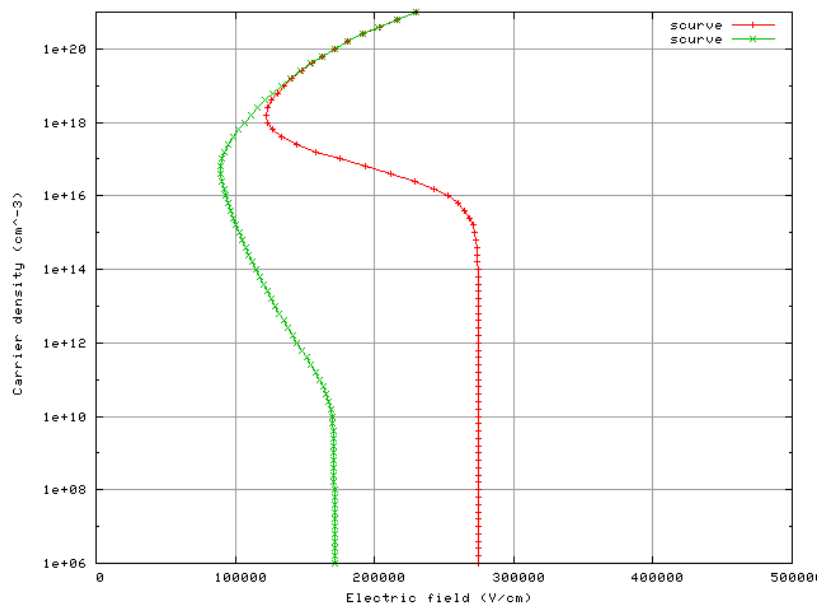

Figure 3 R-curves for two continuum mechanisms, the old mechanism (red) and the new mechanism (green).

The results shown in these figures show that filamentary breakdown occurs at fields that are approximately $100 \mathrm{kV} / \mathrm{cm}$. Such fields are much higher than the lock-on field in GaAs.

An important reason for this similarity is that in both cases the breakdown field is determined by the Maxwellian distribution. We call this field associated with Maxwellian distribution function the Maxwellian field.

In some cases, the k-space calculations have revealed a lower breakdown field than the Maxwellian field. This fact is an important motivation for a new mechanism.

\section{FUTURE WORK}

\section{A. A new lock-on mechanism}

The comparison with lock-on data strongly suggests that the present calculations, even if refined, will not explain lock-on.

At this point we reject our earlier hypothesis that the lock-on field is controlled by the Maxwellian field. This conclusion is based on the large disagreement with the empirical field and also the fact that the k-space calculations for other materials have revealed a lower field.

At this point we make a new hypothesis about a lock-on mechanism. We hypothesize that improved k-space calculations may reveal this field. We recognize that our previous k-space calculations made severe approximations about the inclusion of carrier-carrier scattering events. The approximations made in these calculations may have led to an incorrect calculation of the R-curve at the breakdown field.

We also speculate that a calculation that incorporates band structure in an improved way will produce a mechanism with a lower breakdown field, corresponding to lock-on. To understand this speculation, we note that the present field is dictated by the intrinsic carrier density at a carrier temperature corresponding to the Joule heating. This is a simple result that is nearly independent of the details of the band structure. In fact, it was first obtained in initial calculations that focused on a model material with very simple band-structure.

An important point is that band-structure details were considered but they affected the results in unpredictable ways due to the severe approximations made to include carrier-carrier scattering effects.

The band-structure shown in Fig. 2 suggests a new mechanism. At sufficiently high carrier density, electrons will populate the $\mathrm{L}$ and $\mathrm{X}$ valleys. At high carrier density, electrons in opposite X-valleys can undergo an intra-band Auger transition. This will create one cold and one hot electron. Subsequently, the hot electron, near the $\Gamma$-point, will undergo conventional band to band impact ionization. The basic idea is that under particular conditions, the intra-band Auger recombination will immediately lead to impact ionization.

This mechanism will lead to impact ionization at fields that are much smaller than the field needed for conventional impact ionization. This field-lowering is enabled by a large density of electrons in the heavy mass secondary minima in the band-structure.

This mechanism will only operate in band-structure for which there is a large density of states (DOS) at these secondary minima. Also, the energy separation between the secondary minima and the band-edge must be large compared with the lattice temperature. Given these conditions, a modest electric field can heat electrons to energies that allow the intra-band transitions. 
An empirical fact consistent with this reasoning is that lock-on has only been found in materials that exhibit the Gunn effect. This fact is consistent with this mechanism because the band-structure described here tends to produce a Gunn effect. In this effect, the carrier velocity rises to large value at modest fields but it then drops at high fields.

Another consistency argument is the fact that the impact ionization rate will be proportional to carrier density squared. This density-dependence corresponds to the enhancement factor assumed in the impact ionization term in the generation-recombination rate $R(n, F)$. This particular term is the cause of the filamentary solutions.

\section{B. Future Calculations}

In future work both $\mathrm{k}$-space and continuum calculations will be performed to examine these ideas. The k-space calculations will explore the new mechanism using improved approximations. These new calculations will include nearby $\mathrm{k}$-points as was done in previous calculations but they will also include the new intra-band mechanism.

These calculations of the rate $R(n, F)$ for all electrical fields and carrier densities are very difficult. The main difficulty arises because inclusion of carrier-carrier scattering makes the problem computationally difficult. Furthermore, band structure for arbitrary points in k-space is necessary. Such information is not available even in the low density limit.

Furthermore, a new continuum mechanism will be developed. Preliminary results show that such calculations lead to a continuum mechanism with a lock-on field in agreement with the experimental field.

Finally, we speculate that ordinary electrical breakdown occurs in materials for which an improved calculation does not produce a breakdown field smaller than the Maxwellian field. We note that in either case, the breakdown will produce current filaments.

\section{SUMMARY}

We have described continuum mechanisms for lock-on and electrical breakdown. We have applied this model to optically triggered electrical breakdown in PCSS devices. We find that the qualitative features can be obtained from these calculations. However, the lock-on field computed does not agree with experiments. We hypothesize that an improved mechanism is necessary. We present a hypothesis about such a new mechanism.

\section{REFERENCES}

[1] J. O'Dwyer, "The Theory of Dielectric Breakdown of Solids," Oxford University Press, London, 1964.

[2] H. Raether, "Electron Avalanches and Breakdown of Gases,” Buttersworths, London, 1964.
[3] A. Rosen and F. J. Zutavern, Eds., "High Power Optically-Activated Solid State Switches," Arctech House, Boston, 1994.

[4] G. M. Loubriel, M. W. O'Malley, and F. J. Zutavern, "Toward pulsed power uses for photoconductive semiconductor switches: closing switches," Proc. 6th IEEE Pulsed Power Conf., (IEEE, NY, 1987), Arlington, VA, 1987, pp. 145-148.

[5] F. J. Zutavern, M. W. O'Malley, and G. M. Loubriel, "Recent developments in opening photoconductive semiconductor switches," Proc. 6th IEEE Pulsed Power Conf., (IEEE, NY, 1987), Arlington, VA, 1987, pp. 577580.

[6] F. J. Zutavern, G. M. Loubriel, M. W. O'Malley, W. D. Helgeson, and D. L. McLaughlin, "High gain photoconductive semiconductor switching," Proc. 8th IEEE Pulsed Power Conf., (IEEE, NY, 1991), San Diego, CA, 1991, pp. 23-28.

[7] K. Kambour, H. P. Hjalmarson, C. W. Myles, "A collective theory of lock-on in photoconductive semiconductor switches," Proc. 14th IEEE Pulsed Power Conf., (IEEE, NY, 2003), Dallas, TX, 2003, pp. 345-348.

[8] K. Kambour, "A theory of lock-on and electrical breakdown," Doctoral Dissertation, Texas Tech University, Lubbock, TX, 2003.

[9] H. P. Hjalmarson, F. J. Zutavern, G. M. Loubriel, A. Baca, L. A. Romero, D. R. Wake, K. Khachaturyan, “An Impact Ionization Model for Optically-Triggered Current Filaments in GaAs", Sandia Report SAND93-3972, 1996. [10] K. Kambour, H. P. Hjalmarson, F. J. Zutavern, A. Mar, C. W. Myles, R. P. Joshi, "Simulation of current filaments in photoconductive semiconductor switches," Proc. 15th IEEE Pulsed Power Conf., (IEEE, NY, 2005), Dallas, TX, 2003, pp. 345-348. 